\title{
Lässt sich die Regel des hl. Benedikt auf das moderne Management anwenden?* Teil 2
}

Peter Stulz

* Beim vorliegenden Text handelt es sich um den zweiten Teil der gekürzten Fassung eines Referates an der Jahrestagung und Mitgliederversammlung des Vereins Katholischer Ärzte der Schweiz (VKAS) in Einsiedeln am 10. März 2007.

Korrespondenz:

Prof. Dr. med. Peter Stulz

Kantonsspital

Klinik für Herz-, Thorax-

und Gefässchirurgie

CH-6000 Luzern 16

peter.stulz@ksl.ch

\section{Der heilige Benedikt als «Leader»}

\section{«Qualis debeat esse abbas -}

Wie der Abt sein soll» [1]

Mit diesen Worten überschreibt Benedikt das erste Abtkapitel: In einer heutigen Stellenausschreibung würde man vom Anforderungsprofil des CEO eines modernen Unternehmens sprechen. Es fällt auf: An erster Stelle steht das «esse», das Sein, und nicht das Können. Damit ist ein entscheidender Hinweis auf die Sicht von Amt und Person gegeben. Es geht nicht zuerst um die Fachkompetenz, sondern um «soft skills», um die menschliche und geistliche Glaubwürdigkeit und um die integre Lebensführung. Benedikts Forderungen an den Abt lassen sich auf vier Kernkompetenzen bzw. Schlüsselqualitäten konzentrieren:

- der Abt soll authentisch sein;

- der Abt soll eine Vorbildfunktion einnehmen;

- der Abt soll fähig sein, Verantwortung zu übernehmen;

- der Abt soll einen angemessenen Umgang mit seiner Autorität pflegen.

\section{Authentizität}

Authentizität ist ein facettenreicher und vieldeutiger Begriff. Was den Abt, den CEO, die Führungsperson schliesslich «authentisch» macht, entzieht sich letztlich der Bestimmtheit einer erschöpfenden Definition. Wir müssen demnach den Begriff umschreiben. Dem Wortsinn nach bedeutet Authentizität: Echtheit, Zuverlässigkeit, Glaubwürdigkeit.

Hören wir Benedikt im Eingangsvers des zweiten Abtkapitels:

«Der Abt, der würdig ist, dem Kloster vorzustehen praeesse dignus est - muss immer bedenken - meminere debet-, wie er genannt wird; als «Oberer` erwahre er diesen Namen durch Taten» (RB 2.1).

Das Schlüsselwort «meminere» lässt mehrere Übersetzungen zu: «daran denken», «sich bewusst erinnern», «im Gedächtnis bewahren», «reflektieren». Dieses «meminere» wiederholt sich in fünf Leitsätzen dieses Abtkapitels, ist damit offensichtlich ein Leit- und Lieblingswort Benedikts.

\section{La Règle de saint Benoît se laisse-t-elle appliquer au management moderne? \\ $2^{\mathrm{e}}$ partie}

La Règle de saint Benoît, rédigée vers I'an 550 de notre ère sur le Mont-Cassin, en des temps troublés par la guerre et à une époque de transition entre l'Antiquité tardif et le début du Moyen Age, est bien plus qu'une simple description de directives sur la vie monastique. La Règle de saint Benoît est un concept de vie et un programme, une règle de vie riche et exhaustive. Dans le jargon moderne de gestion d'entreprise, on désignerait cette «Règle» comme étant la charte programmatique du développement de l'organisation de l'Ordre des bénédictins.

Dans la société moderne, presque toutes les personnes vivent leur vie professionnelle au sein d'organisations, de communautés, d'institutions. Comme peu auparavant dans I'histoire, nous avons aujourd'hui énormément de personnes investies de facto de tâches directionnelles et cette tendance s'accentuera à l'avenir, dans les services, l'information et la science. Les exigences, en parallèle, augmenteront considérablement. La plupart d'entre nous devront «gérer», qu'ils le veulent ou non, de manière consciente ou non, d'autres personnes, collaboratrices et collaborateurs, collègues, chefs, ou tout simplement nous-mêmes. Nous serons, sans exception, tous concernés par la «gestion» et devrons, que nous le voulions ou non, trouver ou chercher un intérêt à cette compétence.

La $1^{\text {re }}$ partie a décrit de manière concise quelquesunes des nombreuses formes de leadership efficace et la $2^{\mathrm{e}}$ partie les compare à la Règle de saint Benoît. 
Die Selbstreflexion, das permanente Sichfragen und Hinterfragen: Bin ich so, wie ich mich präsentiere auf der Bühne, der Arena des Lebens? das ständige Sinnieren über die permanent vorhandene Kluft zwischen Sein und Schein - dieses «memor semper»-scheint der Königsweg zur Authentizität zu sein. Leben und Lehren, Wort und Tat müssen sich decken, so will es Benedikt.

Die Quelle der Überzeugungskraft eines Führungsverantwortlichen liegt in der Bedeutungslosigkeit seiner eigenen Person bezogen auf die Aufgabe, die er im Unternehmen einnimmt. Wenn ein Vorgesetzter das auch wirklich tut und danach lebt, ist das für die Mitarbeiter ein kaum zu übersehendes Signal, dass er meint, was er sagt. Glaubwürdig - eben authentisch - ist der, der lebt, was er sagt. Für glaubwürdig gehalten zu werden ist eine sittliche Auszeichnung für einen Menschen. Nur narzisstische Menschen verleihen sich selbst Glaubwürdigkeit. Ob schliesslich ein Mensch glaubwürdig ist, kann er selbst nicht bewerten. Das sagen ihm seine Mitarbeiter/Mitbrüder. Glaubwürdig sein bedeutet mitunter das Erkennengeben und die Einsicht ins eigene Innere, das Loslassen eigener Persönlichkeitselemente (personal leadership / overcoming self-limiting beliefs).

Eine Führungspersönlichkeit, die die tieferen Gründe des eigenen Tuns nicht kennt, wird Mühe haben, einem Team vorzustehen. «Durchs Leben, nicht durchs Reden sichtbar machen» lautet bereits der Rat aus der Bibel. Wer glaubt, Sachverhalte seien losgelöst von subjektivem Empfinden oder spirituellen Vorstellungen zu behandeln, bekommt auf dem Parkett der Chefetagen, dem Dschungel des mittleren Kaders und der Welt der ausgebrannten niederen Chargen täglichen Anschauungsunterricht, dass eher das Gegenteil richtig sei.

\section{Vorbildfunktion}

«Der Abt zeigt eher durch Taten (factis) als mit Worten, was gut ist und heilig» (RB 2.12).

Benedikt setzt hohe Ansprüche an die Vorbildfunktion des Abtes. In gleich zwei Leitsätzen will Benedikt «Taten» (factis) seines Abtes sehen und nicht bloss Worte hören. Diese Aufgabe setzt voraus, dass der Abt in der Gemeinschaft lebt und sich dieser stellt. Der Chef steht mitten unter seinen Mitarbeitern. Ein echter Leader führt nicht vom Chefbüro aus, sondern inmitten seiner Crew: «Management by walking around and speaking together» ist durchaus benediktinisch.

Führen heisst Werte vorleben. Die Führungskräfte sind die Gestalter einer Unternehmenskultur. Sie richten ihre ganze Tätigkeit nach den
Grundwerten des Unternehmens aus und leben diese den Mitarbeitern vor. Die unternehmerischen Grundwerte - bei Benedikt Christus nachzufolgen - geben der Tätigkeit jedes Mitarbeiters Sinn und vermitteln Sicherheit, die unternehmerisches Denken und Handeln ermöglicht (create value for the entire organisation).

\section{Verantwortung}

Die Aufgabe, eine Verantwortung zu übernehmen, fordert Benedikt von seinem Abt mit Vehemenz:

«Der Abt bedenke immer (meminere debet semper abbas ...), was er ist; er bedenke, was für einen Namen er trägt (meminere quot dicitur); und er wisse: «Wem man viel anvertraut hat, von dem wird man um so mehr fordern»» (RB 2.30).

Die Klostergemeinschaft, aber auch die Betriebsöffentlichkeit ist der Prüfstein für Leadership: Heutige Ordensleute stellen hohe Anforderungen an ihre Äbte, und Mitarbeiter eines Betriebes, eines Spitals, verlangen dasselbe von ihren Chefs. Benedikt erinnert den Abt unaufhörlich daran, welche Gewissenhaftigkeit, welches Feingefühl, welcher Gerechtigkeitssinn von ihm gefordert wird. Er schärft ihm immer wieder ein, dass er am Tag des Jüngsten Gerichtes dafür wird geradestehen müssen, dass seine Mönche wirklich finden konnten, was sie im Kloster suchten:

«Der Abt wisse: Wer die Leitung von Seelen übernimmt, muss bereit sein, Rechenschaft abzulegen» (RB 2.37).

«Für so viele Seelen, als er für Brüder verantwortlich ist, für ihre gesamte Zahl, das wisse er ganz sicher, ist er am Tag des Gerichts dem Herrn Rechenschaft schuldig, dazu ohne Zweifel auch für seine eigene Seele» (RB 2.38).

«Auch wisse der Abt, dass die Schuld auf den Hirten fällt, wenn der Hausvater (pater familias) bei seinen Schafen einen Missertrag feststellt - oder etwas freier formuliert: ¿Es soll den Hirten treffen, wenn die Herde mager ist»» (RB 2.7) (Swissair-Debakel).

Benedikt geht mit seinen Äbten also sehr hart ins Gericht. Die Verantwortung des Abtes ist umfassend, unteilbar, persönlich. Verantwortung ist nicht eine Sache von Talent oder Begabung, sondern sie besteht darin, für das, was man tut - und gelegentlich auch für das, was man zu tun versäumt hat-, einzustehen und nicht jeden Fluchtweg aus der Verantwortung zu nützen, der sich bietet. Bedauerlicherweise gibt es gerade in den Organisationen und Unternehmen unserer Gesellschaft viele und manchmal recht raffinierte Fluchtwege aus der Verantwortung. In seinem neuen und sehr empfehlenswerten Buch - «Die Kultur des Neuen Kapitalismus» [2] - lenkt der amerikanische Soziologe Sennett das Augen- 
merk auf die schleichende Auflösung der Verantwortlichkeit - oder auf organisierte Verantwortlichkeit! Wer nicht zu seiner Verantwortung steht, ist kein Leader, allenfalls ein Karrierist. Die Menschen werden sich der Macht beugen, die de facto aus der Position des Vorgesetzten resultiert, insbesondere jene Angestellten, die keine andere Option haben. Aber sie werden der Führungsperson keine Gefolgschaft leisten.

\section{Autorität}

Benedikt stattet den Abt mit grosser Autorität aus. Der Abt sei ein Lehrer. Der Abt lehrt das Gesetz Christi und ist ihm rechenschaftspflichtig, wie wir bereits gehört haben. Der Abt weiss die Regel; er muss sie auf die jeweilige Situation, auf den jeweiligen Einzelfall anwenden. Also braucht er Ermessensspielraum und Entscheidungsgewalt. Der Grund für seine unbestreitbare Autorität liegt letztlich darin, dass er im Kloster die Stelle Christi einnimmt. Benedikt lässt also nicht den geringsten Zweifel daran, wer das letzte Wort haben muss:

«Wer also den Namen 〈Abt annimmt, muss seine Jünger mit doppelter Lehre leiten: D.h.: Er zeige eher durch Taten als mit Worten, was gut und heilig ist. Den fähigeren Jüngern lege er Gottes Gebote in Worten dar, den Hartherzigen aber und den Einfältigeren veranschauliche er die Weisungen Gottes durch sein Beispiel. Hat er seine Jünger gelehrt, dass etwas verboten ist, zeige er auch durch sein Beispiel, dass man es nicht tun darf» (RB 2.11-13).

Dieser Leitsatz enthält Benedikts Vorstellung einer umfassenden Lehrfunktion des Abtes (manager as learner and teacher) - ist Leadershipfunktion. Einmal mehr wird auch die Vorbildfunktion des Abtes im Vorleben von Werten angesprochen.

Benedikt spricht weiter vom Abt als Hirten. Die Funktion des Hirten umfasst das Hüten seiner Schafe, das Umsorgen seiner Schafe, nicht das Beherrschen und Verfügen. Diese Hirtenfunktion eines $\mathrm{CEO}$ hat sich auch in modernen Managementbüchern niedergeschlagen: «Der Qualitätsmanager figuriert als guter Hirte, er weiss, was die ihm Anvertrauten brauchen, und ist stets auf ihr Wohl bedacht, auf das keines verlorengehe. Durch diese gleichermassen auf den einzelnen wie auf die gesamte Herde gerichtete Sorge versichert er sich Loyalität, steigert ihre Leistungen und vermag sie in fruchtbare Weidegründe zu führen.» Diese Aussage eines zeitgenössischen Managerexperten entspricht wortgetreu Benedikts Autoritätsverständnis [3].

Der Begriff «Autorität» leitet sich etymologisch von «augere» ab, was «wachsen machen, vergrös- sern» bedeutet. Der Abt soll den Lebensraum dafür schaffen (augere), wo der einzelne wachsen, sich entfalten, sich verwirklichen kann - das ist Autorität. Der Abt führt nicht autoritär; er ist eine Autorität, die Menschen um sich vereint, die ihre individuelle Persönlichkeit auch am Arbeitsplatz weiterentwickeln, die nicht nur als Arbeitskräfte dienen, sondern als Mitverantwortliche mittragen möchten. «Der Abt sei nicht ängstlich um seine Autorität besorgt», schreibt Benedikt, was heisst: Der Abt soll nicht so sehr aufgrund seiner formalen Stellung wirken als vielmehr mit dem Gewicht seiner ganzen Persönlichkeit.

\section{Quintessenz benediktinischer Führungsweisheit: die Liebe}

«Liebe zu den Menschen»- das ist Benedikts Richtschnur in seinem Führungsverhalten. Die genaue Durchsicht des zweiten Abtkapitels Kapitel 64: Von der Einsetzung des Abtes - lässt die Liebe als Benedikts Leitvokabel auftreten.

Wie Benedikt den Weg zur Liebe findet, ist sehr eindrücklich in dessen «Biographie» durch Papst Gregor den Grossen im Buch II der Dialoge beschrieben und kommentiert [4]. Benedikt vollzieht in der letzten Begegnung mit seiner Schwester Scholastika durch sie den letzten Schritt christlicher Vollendung. Benedikt kommt in dieser Begegnung vom Gesetz hin zur Liebe.

Diese Liebe scheint auf den ersten Blick gegen das Gesetz zu stehen. Scholastika bittet ihren Bruder darum, mit ihr ein nächtliches geistliches Gespräch zu führen und die «Regel» zu brechen, sich am Abend nicht ins Kloster zurückzuziehen. Es ist das erste und einzige Mal, dass sie eine solche Bitte äussert, denn sie weiss um das Ende ihres Lebens. Sie will mit Benedikt gleichsam ihre Totenvigil feiern, eine durchwachte Nacht des Gebetes angesichts des unmittelbaren Todes. Benedikt erkennt die Situation nicht. Er beharrt auf dem Gesetz, auf seiner «Regel» - seinem Lebensmodell! Erst das plötzlich hereinbrechende Gewitter, mit dem der Herr auf das Gebet der Scholastika antwortet, macht ihm eine Rückkehr unmöglich und ist ein Zeichen Gottes (siehe Abbildung).

Es geht nicht darum, die Regel zu unterlaufen, dazu ist der Anlass zu einmalig und zu grundsätzlich. Benedikt muss lernen, dass es Situationen gibt, wo das Gesetz ausser Kraft gesetzt wird - um der Liebe willen. Erst in der Begegnung mit seiner Schwester reift Benedikt zu jener Vollendung im Geiste des Evangeliums heran, die für den vollkommenen Mönch unerlässlich ist: zur Erkenntnis, dass die Liebe über allem steht. Scholastika verwirklicht in ihrer Person, was am Ende 
Abbildung 1

Die heiligen Geschwister Benedikt und Scholastika. Holzschnitt von P. Karl Stadler OSB, Engelberg.

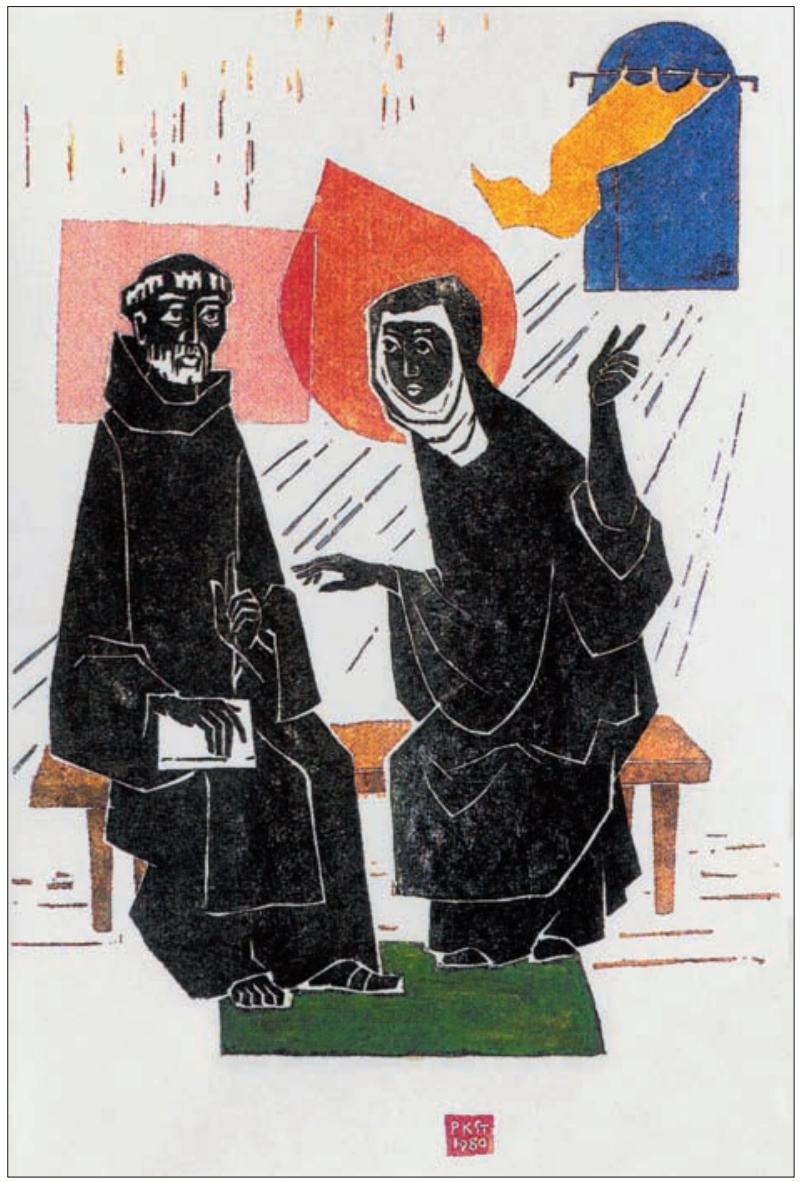

des Prologs der Benediktinerregel als Ziel des monastischen Lebens formuliert ist:

«Wer aber im klösterlichen Leben und im Glauben voranschreitet, dem weitet sich das Herz (dilatatio cordis), und mit der unsagbaren Freude der Liebe eilt er voran auf dem Weg der Gebote Gottes!» (RB Pro$\log 49)$.

Der Wille zu einer klaren Führung, zu einer humanen Führung muss von der Liebe beseelt sein.

$\mathrm{Zu}$ voll und zu umfänglich ist das Phänomen Liebe, um in wenigen Worten eingefangen $\mathrm{zu}$ werden. Was in Benedikts Worten klar durchschimmert, ist die Tatsache, dass wir Liebe brauchen. Gerade in der heutigen Zeit, wo jeder seinen eigenen Weg geht und glaubt, auf den Bezug $\mathrm{zu}$ anderen Menschen verzichten zu können, brauchen wir sie mehr denn je.

Im zweiten Abtkapitel umschreibt Benedikt in einfachen Worten, knapp und prägnant formuliert, was er unter Liebe versteht: Liebe als misericordia - als Barmherzigkeit -, Liebe als caritas - als Hochachtung, Wertschätzung, Dienstbe- reitschaft für das Ganze. Unter «lieben» als Verb versteht Benedikt diligare - hochachten, schätzen -, lieben als prudenter agere - klug handeln -, lieben als prodesse - fördern. Dazu die entsprechenden Leitverse aus Kapitel 64:

«Der Abt sei selbstlos, nüchtern, - et misericordiam barmherzig, - et semper superexaltet misericordiam iudicio - und das Erbarmen übertreffe immer das Richten» (RB 64.9-10).

«Der Abt hasse das Böse - et diligat fratres - und liebe die Brüder» [i.S. von hochschätzen] (RB 64.11). «Der Abt darf keine Fehler wuchern lassen, - sed prudenter et cum caritate ea amputet - sondern er schneide sie klug und liebevoll heraus» (RB 64.14). «Muss der Abt zurechtweisen, so gehe er klug vor prudenter agat - und tue nicht zuviel des Guten, damit das Gefäss nicht zerbricht» (RB 64.12).

Diese vier Leitsätze sind exemplarisch für Benedikts Führungsverhalten in Situationen, in denen er mit schwierigen Mitbrüdern zu kämpfen hat und in denen er fehlerhaftes Verhalten kraft seines Amtes korrigieren muss. Korrekturmassnahmen, die auf Liebe und Barmherzigkeit, aber auch auf Wertschätzung des Mitmenschen beruhen, sind nicht Strafe, sondern Therapie und Hilfe, die richtige Marschroute wiederum aufzunehmen und nicht auf Abwege ohne Rückzugsmöglichkeiten zu gelangen.

In diesem Kontext muss ich noch einmal auf die Autorität zu sprechen kommen. Wie üben wir als Führungsverantwortliche Autorität aus? Das ist doch die entscheidende Frage im Hinblick auf eine humane Unternehmenskultur. Benutzen wir unsere Autorität zur Abwertung oder zur Aufwertung eines Menschen? Davon hängt das Arbeitsklima ab, davon hängt auch der Erfolg jedes Vorgesetzten ab. «In Liebe dienen, nicht mit Macht beherrschen», so wahrt Benedikt die Autorität des Abtes.

Wenn man diese Leitverse überdenkt, beginnt man zu begreifen, was für Benedikt wahre Autorität ist - nämlich eine Instanz, die weiterhilft, unterstützt, ermutigt, ermahnt und jederzeit den Fortschritt und das Wohl der Menschen im Auge hat, die ihm anvertraut sind.

Die folgenden zwei Leitverse sind für mich persönlich wohl die beiden wichtigsten Schlüsselsätze einer humanen Führung:

«Et studeat plus amari quam timeri - der Abt versuche, mehr geliebt als gefürchtet zu werden» (RB 64.15).

Der Abt soll sich nicht einschleichen oder beliebt machen als «Kumpel», sondern prüfen, ob seine innere Hochachtung der eines liebenden Vaters («Patron» im ursprünglichen guten Sinne) entspricht. Für den Abt bedeutet diese Erwartung eine bleibende Herausforderung. 
«Magis prodesse quam praeesse - der Abt wisse, dass es für ihn wichtiger ist, mehr vorzusehen als vorzustehen» (RB 64.8).

Das Wortspiel «Magis prodesse quam praeesse» lässt jedoch noch eine andere Deutung zu. Der versierte Altphilologe Abt em. Gregor Holzherr [1] spricht beim Wort «prodesse» von einem Hapaxlegomenon, d.h. von einem nur einmal belegten, in seiner Bedeutung oft nicht genau zu bestimmenden Wort einer nicht mehr gesprochenen Sprache. Deshalb billigt Pater Holzherr dem Wort «prodesse» auch die Bedeutung von «fördern», «nützen», «nützlich sein» zu:

«Magis prodesse quam praeesse - der Abt wisse, dass es für ihn wichtiger ist, mehr zu fördern, zu «ützen` als vorzustehen» (RB 64.8).

Welch tiefsinniger Merksatz, in dem der dienende Charakter des Amtes eines Führungsverantwortlichen einer Klostergemeinschaft, einer Institution herausgehoben wird!

Dies ist ein komplementärer Gesichtspunkt zum Gehorsam der Mitbrüder, der Mönche. Ge-Horchen ist eine intensive Weise des Hörens bedeutet also, ein aufmerksames Ohr zu haben. In diesem Sinne kann Gehorsam nie nach dem Muster politischer Machtstrukturen verstanden werden. Weder monarchische noch demokratische (es kann jeder machen, was er will) Führungsstrukturen schweben Benedikt vor, sondern eine von Liebe getragene Dienstauffassung. Paragraphenreiterei, starres Festhalten am Legalismus sind Benedikt fern. Benedikt ist kein Bürokrat im Sinne des traditionellen Managers, Benedikt ist ein hochmoderner Leader.

Lassen Sie mich als Mediziner in einer Schlusspointe zur «Herzmedizin» zurückkehren - zum Herz als Symbol der Liebe. Wenn Benedikt jetzt hier unter uns Medizinern wäre - er pflegte ja durchaus ein sehr gutes Verhältnis zur «Medizin» seiner Zeit -, würde er uns ein vertrautes Instrument - ein Stethoskop - als Führungsinstrument überreichen mit dem Hinweis: Der Führungsverantwortliche sollte nicht nur ein «Könner in der Auskultation von Galopptönen und pathologischen Geräuschen sein, sondern ebenso die Kunst einer noch anspruchsvolleren Auskultation verborgener Schwingungen im Menschen beherrschen, also begabt im Hören mit dem Stethoskop, aber auch mit den Ohren des Herzens» (nach Prof. Frank Nager).

Dieser abschliessende Gedanke bringt uns zurück zum allerersten Leitvers im Prolog, in dem Benedikt seine Regel, seinen Lebensentwurf mit den Worten eröffnet: «Obsculta, o fili, praecepta magistri, et inclina aurem cordis tui [...]»-
«Höre, mein Sohn [...], und neige das Ohr deines Herzens [...]», ausgerichtet auf die Beziehung zu Christus und auf ein Leben nach seinen Weisungen. Der Kreis ist geschlossen.

\section{Epilog}

Was an der «Regel» des heiligen Benedikt fasziniert:

1. Die Regel zeichnet sich aus durch Kürze, Klarheit und Übersichtlichkeit. Sie lässt auch Raum für Entscheidungsfreiheit und Verantwortung. Die Sprache ist einfach, klar, verständlich, bodenständig, nicht abgehoben, zeugt von Lebenserfahrung. Benedikt zeigt eine ausgesprochene Tendenz zur Kürze und verzichtet auf romantisches Geschwätz (beispielsweise) heutiger Managergurus. Benedikt ist ein Mensch, der von der Begegnung mit anderen Menschen lebt, von ihrer Begleitung, von ihrer Zuwendung, von ihrer Korrektur. Benedikt flüchtet sich nicht in abstrakte Gespräche. Er liebt die konkreten Dinge und sieht die Menschen, wie sie sind, alltäglich und greifbar. Benedikt sagt, was er denkt.

2. Das Leben nach der RB verbindet das Geistliche/Spirituelle mit dem Irdischen. So ist das «ora et labora» zu einer elementaren Maxime für das Monastische schlechthin geworden. Hans Urs von Balthasar schreibt in seinem Buch «Die grossen Ordensregeln»: «Benedikt hatte mit seinem Grundsatz «ora et labora eine für die damalige Zeit geradezu revolutionäre Verbindung von meditativen, kontemplativen Anliegen mit aktiven, produktiven Elementen geschaffen und so eine abendländischer Mentalität gemässe Form mönchischer Frömmigkeit gefunden. Wenngleich er selber und auch immer wieder in der Einsamkeit Gott suchte, distanzierte sich Benedikt mit seiner Regel doch von der extremen Askese (etwa der Kartäuser) und dem Einsiedlertum, mit denen die ersten christlichen und vorab im Osten des Mittelmeerraumes stark verbreiteten Mönche der Welt und ihren Versuchungen zu entfliehen suchten.»

Seit Jahrhunderten weist man Benedikt das Motto «ora et labora» zu. Es steht nicht in diesem Wortlaut in der «Regel», beschreibt aber etwas Wesentliches von Benedikts Spiritualität. Das geistliche Leben beinhaltet nicht nur Beten, sondern die «geerdete» (P. Anselm Grün) Spiritualität zeigt sich insbesondere auch an der Art der Arbeit. Die Verbindung von Gebet und Arbeit deutet nicht nur auf das richtige Mass dieser beiden Pole unseres Lebens, sondern meint bei Benedikt die in- 
nere Durchdringung dieser beiden Lebenselemente. Damit wird deutlich, dass die Arbeit weder isoliert gesehen wird noch sich verselbständigen darf. Die Leistungsfähigkeit, d.h. die Bereitschaft, sich durch Arbeit - welche auch immer - fordern zu lassen, ist ein wesentliches Element benediktinischer Spiritualität. Die Arbeit erhält demnach eine spirituelle Aufwertung.

Die RB ist ein Plädoyer für das Ethos der Arbeit, ein Aufruf zu verantwortlicher Arbeit. Die Arbeitspflicht - immer in Anbetracht der Möglichkeiten und Fähigkeiten der einzelnen Mönche - gehört zu den Grundelementen des gemeinsamen Lebens. Die Anweisung zur Arbeit ist zugleich eine Korrektur gegenüber der von manchen Mönchskreisen propagierten Idee, die Aufgabe des asketischen Lebens sei allein Gebet und Lesung!

3. Wir leben in einer Zeit der Unordnung, des Chaos, der Relativität, des Individualismus bis hin zur Perversion. Die RB gibt eine Anleitung dafür, den Wechsel von Anspannung und Entspannung, von Aussenorientierung und Innerlichkeit bewusst zu leben. Ordnung, klare Struktur des Tages, Umgang mit der Zeit, Wichtigkeit der Ordnung in der Persönlichkeit, Reduzierung auf Wesentliches sind Hinweise bzw. Impulse für das persönliche «Time Management».
4. Die einschlägigen Kapitel - für unsere Betrachtung die beiden «Abtkapitel» 2 und 64 der 1500jährigen RB erweisen sich auch heute noch als hochaktuelle Managementprinzipien. Wie bereits erwähnt soll man die «Regel» nicht auf ein Managementmanual reduzieren! Das wäre eine einseitige Betrachtungsweise. Es werden Führungskonzepte vermittelt, bei denen die fachliche Kompetenz um ethische und spirituelle Aspekte erweitert wird.

\section{Literatur}

1 Die Benediktsregel. Eine Anleitung zu christlichem Leben. Der vollständige Text der Regel, übersetzt und erklärt von P. Georg Holzherr OSB, em. Abt von Einsiedeln. 6., völlig überarbeitete Auflage. Freiburg i. Ü.: Paulusverlag; 2005. RB = Regula Benedicti, erste Zahl = Kapitel (Prolog, 1-73), zweite Zahl $=$ Leitvers.

2 Sennett R. Die Kultur des Neuen Kapitalismus. Berlin: Berlin Verlag; 2005.

3 Bröckling U. Totale Mobilmachung. Menschenführung im Qualitäts- und Selbstmanagement. In: Bröckling U, et al. (Hrsg.). Gouvernementalität der Gegenwart. Studien zur Ökonomisierung des Sozialen. Frankfurt am Main: Suhrkamp; 2000. S. 131-67.

4 Gregor der Grosse. Der hl. Benedikt. Buch II der Dialoge; lateinisch/deutsch. Herausgegeben im Auftrag der Salzburger Äbtekonferenz. Erzabtei St. Ottilien: EOS Verlag; 1995. Kapitel 33, S. 189-92, Kommentar S. 70-1. 